\title{
Correction to: Isogeometric hyperelastic shell analysis with out-of-plane deformation mapping
}

\author{
Kenji Takizawa ${ }^{1} \cdot$ Tayfun E. Tezduyar $^{2,3} \cdot$ Takafumi Sasaki $^{1}$
}

Published online: 19 November 2019

๑) Springer-Verlag GmbH Germany, part of Springer Nature 2019

\section{Correction to:}

\section{Computational Mechanics (2019) 63:681-700 https://doi.org/10.1007/s00466-018-1616-3}

In the original publication [1], a term was missing in Eq. (99). The correct form is

$$
\delta \overline{\mathbf{g}}^{\gamma}=\left(\bar{g}^{\gamma \delta} \mathbf{n n}-\overline{\mathbf{g}}^{\delta} \overline{\mathbf{g}}^{\gamma}\right) \cdot \delta \overline{\mathbf{g}}_{\delta} .
$$

Consequently, Eqs. (97) and (98) also had missing terms. The correct forms are

$$
\begin{aligned}
\delta_{a} \delta_{b} \bar{\kappa}_{\alpha \beta}= & \left(\delta_{a} \overline{\boldsymbol{\Gamma}}_{\alpha \beta}-\left(\overline{\boldsymbol{\Gamma}}_{\alpha \beta} \cdot \overline{\mathbf{g}}^{\gamma}\right) \frac{\partial \delta_{a} \overline{\mathbf{x}}}{\partial \xi^{\gamma}}\right) \cdot \overline{\mathbf{g}}^{\gamma}\left(\mathbf{n} \cdot \delta_{b} \overline{\mathbf{g}}_{\gamma}\right) \\
& +\delta_{a} \overline{\mathbf{g}}_{\gamma} \cdot \mathbf{n}\left(\overline{\mathbf{g}}^{\gamma} \cdot \delta_{b} \overline{\boldsymbol{\Gamma}}_{\alpha \beta}+\overline{\boldsymbol{\Gamma}}_{\alpha \beta}\right. \\
& \left.\cdot\left(\bar{g}^{\gamma \delta} \mathbf{n n}-\overline{\mathbf{g}}^{\delta} \overline{\mathbf{g}}^{\gamma}\right) \cdot \delta_{b} \overline{\mathbf{g}}_{\delta}\right) \\
= & \left(\delta_{a} \overline{\boldsymbol{\Gamma}}_{\alpha \beta}-\left(\overline{\boldsymbol{\Gamma}}_{\alpha \beta} \cdot \overline{\mathbf{g}}^{\gamma}\right) \frac{\partial \delta_{a} \overline{\mathbf{x}}}{\partial \xi^{\gamma}}\right) \cdot \overline{\mathbf{g}}^{\gamma}\left(\mathbf{n} \cdot \delta_{b} \overline{\mathbf{g}}_{\gamma}\right) \\
& +\left(\delta_{b} \overline{\boldsymbol{\Gamma}}_{\alpha \beta}-\left(\overline{\boldsymbol{\Gamma}}_{\alpha \beta} \cdot \overline{\mathbf{g}}^{\delta}\right) \frac{\partial \delta_{b} \overline{\mathbf{x}}}{\partial \xi^{\delta}}\right) \cdot \overline{\mathbf{g}}^{\gamma}\left(\mathbf{n} \cdot \delta_{a} \overline{\mathbf{g}}_{\gamma}\right) \\
& +\left(\mathbf{n} \cdot \delta_{a} \overline{\mathbf{g}}_{\gamma}\right) \bar{b}_{\alpha \beta} \bar{g}^{\gamma \delta}\left(\mathbf{n} \cdot \delta_{b} \overline{\mathbf{g}}_{\delta}\right) .
\end{aligned}
$$

We note that the missing term is on the left side of the iteration equations, and the computations were performed without the

The original article can be found online at https://doi.org/10.1007/ s00466-018-1616-3.

Kenji Takizawa

Kenji.Takizawa@tafsm.org

Tayfun E. Tezduyar

tezduyar@tafsm.org

1 Department of Modern Mechanical Engineering, Waseda University, 3-4-1 Ookubo, Shinjuku-ku, Tokyo 169-8555, Japan

2 Mechanical Engineering, Rice University - MS 321, 6100 Main Street, Houston, TX 77005, USA

3 Faculty of Science and Engineering, Waseda University, 3-4-1 Ookubo, Shinjuku-ku, Tokyo 169-8555, Japan term. However, we confirm that the inclusion of the term does not change the results presented in the article. We also note that in a subsequent publication [2], we had equations similar to Eq. (99), but in correct form.

Equation (99) had a missing term because Eq. (142) in Appendix $\mathrm{C}$ had a missing term. We now provide the corrected Appendix $\mathrm{C}$ in its entirety.

\section{Appendix C: Variation of the contravariant basis vector}

Here we show that $\delta \mathbf{g}^{\gamma}$ can be expressed as

$\delta \mathbf{g}^{\gamma}=\left(g^{\gamma \delta} \mathbf{n n}-\mathbf{g}^{\delta} \mathbf{g}^{\gamma}\right) \cdot \delta \mathbf{g}_{\delta}$.

We start with the transformation from the contravariant basis vectors to the covariant basis vectors:

$\mathbf{g}_{\alpha}=g_{\alpha \delta} \mathbf{g}^{\delta}$.

We take the variation of both sides:

$\delta \mathbf{g}_{\alpha}=\delta g_{\alpha \delta} \mathbf{g}^{\delta}+g_{\alpha \delta} \delta \mathbf{g}^{\delta}$,

and from that obtain

$g_{\alpha \delta} \delta \mathbf{g}^{\delta}=\delta \mathbf{g}_{\alpha}-\delta g_{\alpha \delta} \mathbf{g}^{\delta}$.

From that and Eq. (11), we obtain

$$
\begin{aligned}
g_{\alpha \delta} \delta \mathbf{g}^{\delta} & =\delta \mathbf{g}_{\alpha}-\left(\delta \mathbf{g}_{\alpha} \cdot \mathbf{g}_{\delta}+\mathbf{g}_{\alpha} \cdot \delta \mathbf{g}_{\delta}\right) \mathbf{g}^{\delta} \\
& =\delta \mathbf{g}_{\alpha}-\delta \mathbf{g}_{\alpha} \cdot \underbrace{\mathbf{g}_{\delta} \mathbf{g}^{\delta}}_{=\mathbf{I}-\mathbf{n n}}-\mathbf{g}_{\alpha} \cdot \delta \mathbf{g}_{\delta} \mathbf{g}^{\delta} \\
& =\delta \mathbf{g}_{\alpha}-\delta \mathbf{g}_{\alpha}+\mathbf{n n} \cdot \delta \mathbf{g}_{\alpha}-\mathbf{g}_{\alpha} \cdot \delta \mathbf{g}_{\delta} \mathbf{g}^{\delta} \\
& =\mathbf{n n} \cdot \delta \mathbf{g}_{\alpha}-\mathbf{g}_{\alpha} \cdot \delta \mathbf{g}_{\delta} \mathbf{g}^{\delta} .
\end{aligned}
$$


Multiplying both sides with $g^{\gamma \alpha}$, we obtain

$\underbrace{g^{\gamma \alpha} g_{\alpha \delta}}_{=\delta_{\delta}^{\gamma}} \delta \mathbf{g}^{\delta}=g^{\gamma \alpha} \mathbf{n n} \cdot \delta \mathbf{g}_{\alpha}-\underbrace{g^{\gamma \alpha} \mathbf{g}_{\alpha}}_{=\mathbf{g}^{\gamma}} \cdot \delta \mathbf{g}_{\delta} \mathbf{g}^{\delta}$.

Thus,

$$
\begin{aligned}
\delta \mathbf{g}^{\gamma} & =g^{\gamma \delta} \mathbf{n n} \cdot \delta \mathbf{g}_{\delta}-\mathbf{g}^{\gamma} \cdot \delta \mathbf{g}_{\delta} \mathbf{g}^{\delta} \\
& =\left(g^{\gamma \delta} \mathbf{n n}-\mathbf{g}^{\delta} \mathbf{g}^{\gamma}\right) \cdot \delta \mathbf{g}_{\delta} .
\end{aligned}
$$

\section{References}

1. Takizawa K, Tezduyar TE, Sasaki T (2019) Isogeometric hyperelastic shell analysis with out-of-plane deformation mapping. Comput Mech 63:681-700. https://doi.org/10.1007/s00466-018-1616-3

2. Sasaki T, Takizawa K, Tezduyar TE (2019) Medical-image-based aorta modeling with zero-stress-state estimation. Comput Mech 64:249-271. https://doi.org/10.1007/s00466-019-01669-4

Publisher's Note Springer Nature remains neutral with regard to jurisdictional claims in published maps and institutional affiliations. 